\title{
Detection of Medication Errors in Primary Care Units through Passive Voluntary Reporting Forms
}

\author{
Rowan W. El-Ghareeb ${ }^{\text {a }}{ }^{*}$, Nagwa A. Sabri ${ }^{a}$, Manal H. El Hamamsy ${ }^{a}$, Amr A. Saad \\ ${ }^{a}$ Clinical Pharmacy Department, Faculty of Pharmacy, Ain Shams University, Cairo, Egypt \\ ${ }^{b}$ Former Head of the Egyptian Pharmaceutical Vigilance Center (EPVC), Egypt
}

\begin{abstract}
The lack of a universally accepted terminology of what constitutes a Medication Error (ME), makes it difficult to report, detect, categorize and prevent MEs. Methodologically, there isn't a complete picture of the incidence and prevalence of MEs. The broad range of ME rates in literature reflects heterogeneity in the study designs and detection methods used. The current study aimed to detect MEs in reports received from Primary Care Units. A retrospective analysis was applied on such reports dated from March to November 2013 and some fatal cases are taken from 2014. All voluntary reports were included, excluded were errors not associated with drug use and pharmaceutical company reports. Eligible reports underwent assessment using predetermined criteria to pick up MEs. The criteria were applicable on 115 reports, in which MEs were detected. 60\% (69/ 115) of ME cases were error cluster while 40\% (46/115) were unknown due to either underreporting, lack of data or poor observation and correlation $(\mathrm{p}<0.05)$. Only $7 \%(8 / 115) \mathrm{p}<0.001$ of the reports were pregnancy cases. Moreover, errors associated with vaccine use accounted for $7 \%(8 / 115)$ of the cases while $93 \%$ accounted for errors from other drug use at $\mathrm{p}<0.001$. Long-term follow-up was needed but not done by the assessors in $41 \%$ (47 of 115) of ME cases at pvalue $=0.05$. Attachments were provided with the reports in $9 \%(10 / 115)$ of the cases while the majority $91 \%(105 / 115)$ were not $(\mathrm{p}<0.001)$. In conclusion, voluntary reporting is a major strategy to prevent MEs by learning from errors reported.
\end{abstract}

Keywords: Medication Errors; Passive Voluntary Reporting; Primary Care Units

*Correspondence | Rowan W El-Ghareeb; Department of Clinical Pharmacy; Faculty of Pharmacy, Ain Shams University, Cairo, Egypt.

Email: r_a_143@hotmail.com

Citation | El-Ghareeb RW, Sabri NA, El Hamamsy MH, Saad AA, 2019. Detection of Medication Errors in Primary Care Units through Passive

Voluntary Reporting Forms. Arch Pharm Sci ASU 3(1): 123-129

DOI: 10.21608 /APS.2019.45300

Online ISSN: 2356-8380. Print ISSN: 2356-8399.

Received 17 February 2019. Accepted 10 April 2019.

Copyright: ${ }^{\circledR} 2019$ El-Ghareeb et al. This is an open-access article licensed under a Creative Commons Attribution 4.0 International License (CC BY 4.0), which permits unrestricted use, distribution, and reproduction in any medium, provided the original author(s) and source are credited.

Published by: Ain Shams University, Faculty of Pharmacy

\section{INTRODUCTION}

According to recent studies, medical errors may account for as many as 251,000 deaths yearly in the United States (U.S), making them the third most important cause of death after heart disease and cancer [1]. According to FDA, among the most common medical errors are medication errors (ME); more than 1.5 million people are harmed annually by ME in hospitals, caring homes, and clinics according to a report from the Institute of Medicine [2]. Although ME is a significant cause of morbidity and mortality, its influence on the public can be 
Selective Small MCL-1 Inhibitors: Novel Agents in Cancer Therapy 
underappreciated [3].

More information is known on errors arising in hospitals than in other health care settings [4]. Every year hundreds of thousands of patients - in the (U.S) alone- experience a drug-related problem but often do not report it [5].

The absence of a universally accepted definition and standardized nomenclature for ME has complicated MEs studying and reporting. As a result, many healthcare professionals get confused when reporting medication incidents. The definition of ME proposed by Dr. Jeffrey Aronson overcomes the shortcomings existed in the other published definitions of ME [6]. Medication Error is defined as "a failure in the treatment process that leads to or has the potential to lead to, harm to the patient" [7]. The definition indicates that the treatment process fell under some achievable benchmark with regard to therapy, case investigation, prevention and that the error may not lead to harm. Thus, the definition provides a chance for the surveillance of medicines [6].

Data collection methods commonly used are incident report review, patient chart review, direct observation methods, trigger tools, and pharmacists' interventions. There are three common approaches taken in the existing reporting systems used to identify adverse drug reactions and medication errors. The first approach involves mandatory reporting to an external entity usually used by some countries that oblige reporting by health care organizations. The second one is internal mandatory reporting with the audit. The third approach involves passive reporting (known as voluntary case reporting) to an external group $[4,8,9]$.

Passive reporting is a major strategy to reduce MEs. It requires the active participation of health care professionals to disseminate a patient safety culture. [8, 9] Moreover, the advantages of voluntary reports are being confidential, low cost and commonly used in a lot of medical settings. [10] The major role of passive voluntary reports is to enhance safety by learning from failures of the health-care system. Weak systems rather than individuals, having common root causes, which can be generalized and corrected [11], provoke errors.

The current study aimed to detect MEs involuntary case reports received from hospitals, pharmacies, health care units, and health centers in order to improve patient safety by learning from system errors and preventing them.

\section{MATERIAL AND METHODS}

\subsection{Study Design}

A retrospective study was conducted on reports received from the primary care units 'mentioned in 2.2' dated from March to November 2013 and additional reports dated in 2014.

\subsection{Setting}

2.2.1. The wards and intensive care units of different hospitals including governmental, teaching, insurance, military and private hospitals in Alexandria, Egypt.

2.2.2. Health care units, health centers, and outpatient clinics in Alexandria, Egypt.

2.2.3. Pharmacies (community and hospital) in Alexandria, Egypt.

\subsection{Eligibility Criteria}

\subsubsection{Inclusion Criteria}

All voluntary case reports from healthcare professionals: physicians, pharmacists, and nurses, as well as from patients and their relative were included.

\subsubsection{Exclusion Criteria}

Pharmaceutical company reports and reports on errors not associated with drug use. 


\subsection{Working Procedure}

2.4.1. Data collection of all the reporting forms received from healthcare professionals: physicians, pharmacists, nurses, or patients and their relatives.

2.4.2. Undertaking data analysis and step-by-step detection.

2.4.3. All reports were assessed for eligibility. After meeting the eligibility criteria, all passive

voluntary reports were included while reports on errors not associated with the use of drugs as well as pharmaceutical company reports were excluded.

2.4.4. All the included reports were evaluated by studying each case separately to pick up those containing medication errors through:

2.4.4.1. Applying the ME definition proposed by Dr. Jeffrey Aronson on the cases 'refer to 1'

2.4.4.2. Checking the doses, interactions and compatibility for all the cases reported.

2.4.4.3. Checking the known pharmacology and adverse reactions of the reported medicines.

2.4.4.4. Assuring that the ordered drug was the one received and administered to the patient at the prescribed dose.

2.4.4.5. Assessing the analyst's (assessor) primary follow-up with special attention to the valuable information added by the reporter during the follow-up.

2.4.5. Dropouts were reports of $\mathrm{ME}$ associated with drug quality problems and lack of reporter's contact information.

2.4.6. The Criteria was applicable to 115 reports.

2.4.7. Determining error clusters, indicating the cases with repetitive patterns of the same error, on multiple persons, in the same place.
2.4.8. Determining $\mathrm{ME}$ in special fields of medicine use in pregnancy (teratogenicity).

2.4.9. Determining $\mathrm{ME}$ associated with vaccine use.

2.4.10. Determining the frequency of the cases where further follow-up was required but was not done by the assessors, further, follow-up is required in case of missing patient outcomes or pregnancy cases with teratogenicity potential that needs long-term follow-up.

2.4.11. Evaluating the number of cases where reporters sent supplementary attachments, case narrative, other comments or additional information for more illustration and case explanation.

\subsection{Statistical Methods}

Statistical analysis was done using SPSS, 2008 Statistical Package for the Social Sciences (SPSS), version 17.0 Chicago, USA: SPSS Inc. Qualitative data were expressed as frequency and percentage. Chi-square test was used to examine the relation between qualitative variables. A p-value $<0.001$ was considered highly significant and $p$-value $<0.05$ was considered significant.

\section{RESULTS}

A retrospective analysis was applied on reports dated from March to November 2013 and additional reports dated in 2014 which were received from primary care units including different hospitals, health care units, health centers, outpatient clinics and pharmacies in Alexandria, Egypt. The reports, which fit the inclusion criteria, were included in the study. Out of 211 reports screened, only 115 reports fulfilled the predetermined criteria.

\subsection{Data Analysis and Findings}

Reporters mentioned that 60\% (69/115) of ME cases were of repeated patterns on multiple 
individuals in the same health care setting, thus were considered to be error cluster while the remaining $40 \%(46 / 115)$ of the cases were unknown if error cluster or not; due to either underreporting, lack of data or poor observation and correlation between the error and its reoccurrence with significant difference at $(\mathrm{p}<0.05)$. Error Cluster is represented in Fig. 1.

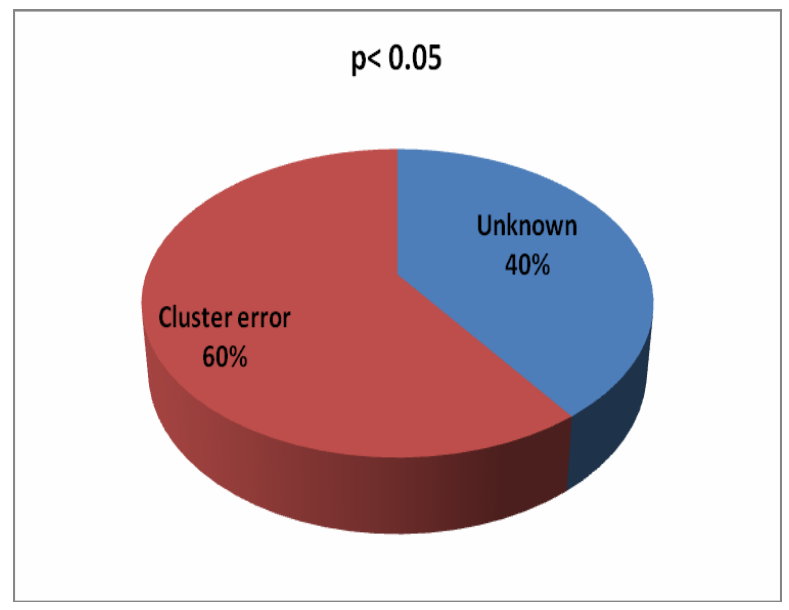

Fig. 1. Error Cluster

Only $7 \%(8 / 115) \mathrm{p}<0.001$ of the reports were pregnancy cases reported during the study time. Pregnancy Cases are illustrated in Fig. 2.

Moreover, errors associated with vaccine use accounted for 8 out of 115 cases (7\%) while 93\% accounted for errors from other drug use with a highly significant difference $\mathrm{p}<0.001$.

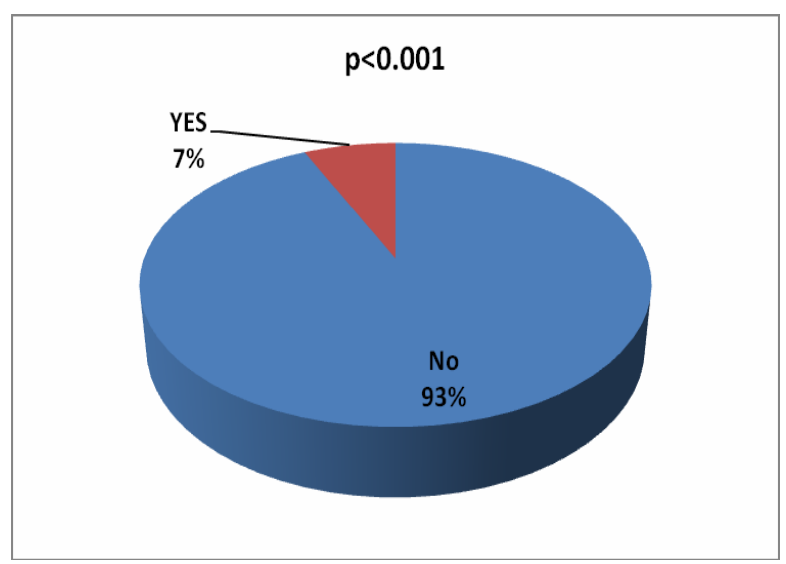

Fig. 2. Pregnancy Cases

Medication Errors from Vaccine Use are represented in Fig. 3.

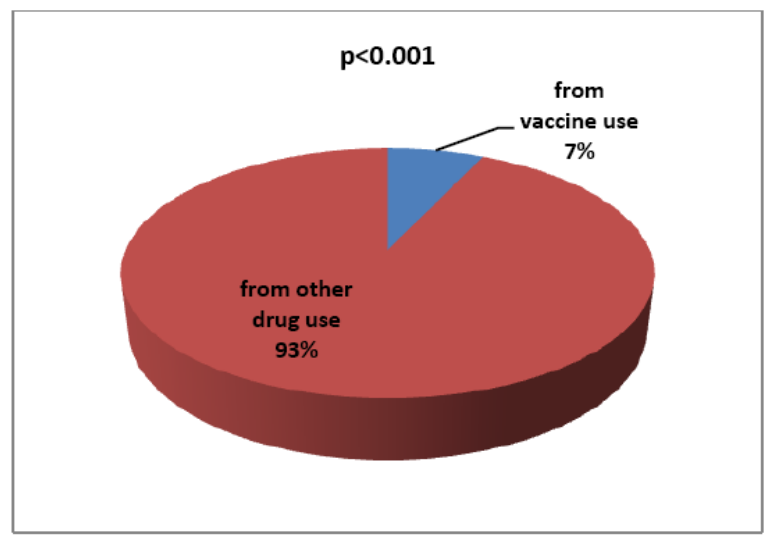

Fig. 3. Medication Errors from Vaccine Use

Further and long-term follow-up was needed but not done by the assessors in $41 \%$ (47 of 115) of ME cases at $\mathrm{p}$-value $=0.05$, including cases of missing patient outcomes and pregnancy cases with teratogenic potential that needs long-term monitoring. Further and Long term Follow-up not done by Assessors is mentioned in Fig. 4.

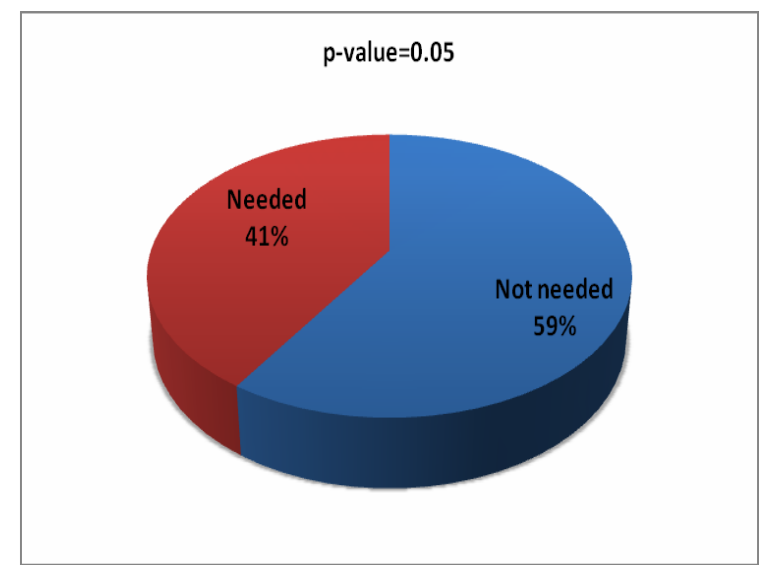

Fig. 4. Further and Long term Follow-up not done by Assessors

Reporters provided attachments with the reports in $9 \%(10 / 115)$ of the cases while the majority $91 \%(105 / 115)$ did not, with highly significant difference $\mathrm{p}<0.001$. Attachments with the $\mathrm{p}<0.001$ from Reporting Form are represented in Fig. 5. 


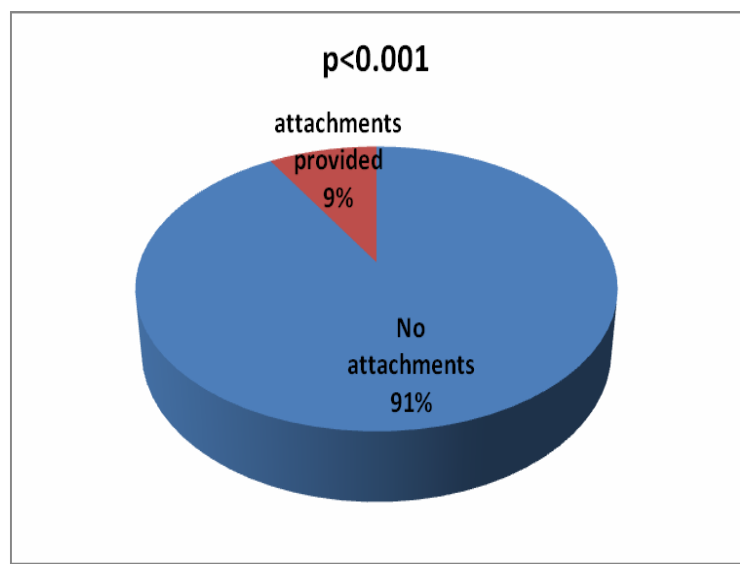

Fig. 5 Attachments with the Reporting Form

\section{DISCUSSION}

One of the first steps in building patient safety is to conduct a retrospective analysis of existing reporting forms in order to pick up MEs as was done in this study. Similarly, eight countries undertook a retrospective study on $\mathrm{ME}$ for the year 2006: Ireland, Iran, Madagascar, Malaysia, Moldavia, Morocco, Nigeria, and Switzerland [12]. Similarly, Milch et al. mentioned that a review of voluntary error reports is an important method to identify MEs [13]. In the present study, it was determined that $60 \%$ (69/ 115) of the reported cases were error cluster indicating the reoccurrence of the same error with several individuals in the same health care setting. Similarly, the Vaccine Adverse Event Reporting System (VAERS) received reports on error clusters as well. This reveals the importance of reporting error cluster in order to prevent the repetitive patterns of such errors, which can be easily generalized among health care settings, therefore monitored, and corrected. In the current study, vaccination errors accounted for 8 out of 115 cases (7\%) which is in agreement with the vaccination error reports sent to VAERS which also accounted for $7 \%$ of total United States VAERS reports in 2000-2013 [14].

While, 40\% (46/115) of ME cases in the current study were unknown whether cluster error or not due to underreporting, lack of data or poor observation and correlation by the reporters. Therefore, the present study emphasizes that cluster errors within the healthcare setting should be observed, studied, monitored and investigated through internal and external reporting in an attempt to find solutions to repeated complains, in agreement with the research results that health care professionals should be aware of MEs repetitive patterns to avoid future errors [15].

In the present study, further and long term follow-up was needed but not done by the assessors in 41\% (47 of 115) of ME cases which was not in accordance with the Guidelines on the Regulation of Therapeutic Products in New Zealand, 2017 as it dictates the necessity to follow up incomplete reports because it is particularly essential for prospective reports of exposure in pregnancy, fatalities or new safety concerns. If the information received from the consumer is incomplete, assessors should try to make direct contact with the consumer or a nominated healthcare provider for more information [16]. The reporting rate of fetal disorders has increased during the last 20 years, from only one report in 1991 to an upper limit of thirty- one reports in 2008 [17]. In the current study, only $7 \%(8 / 115) \mathrm{p}<0.001$ of the reports were pregnancy cases. This indicates the importance of reporting the drug-induced teratogenicity.

In the study of Anderson et al., those who participated found it hard to fit all the necessary information onto the reporting form, meanwhile they were generally not keen on providing data on a separate sheet as suggested by the form [18]. The same was shown in this study where attachments were provided with the reports in only $9 \%(10 / 115)$ of the cases while the majority $91 \%(105 / 115)$ of the cases was submitted without attachments. 


\section{Limitations}

Similar to other voluntary reported data, the information collected may be subject to bias due to underreporting, poor documentation and reporter's individual decision on which errors to be reported. Moreover, interobserver variability in analyzing the cases and the failure of the assessors to follow-up the cases may lead to inaccurate estimations. Furthermore, the calculated rates of medication errors indicate the reporting rates more than the actual occurrence rates; however, it is a helpful tool to detect system weakness which requires improvement. The study was conducted in primary care units in Alexandria, Egypt, thus affects the generalizability of the results.

\section{Conclusion}

Voluntary reporting helps create a team approach to enhance patient safety and prevent ME risks. Encouraging healthcare providers on efficient reporting is highly demanded as well as increasing the awareness of the assessors on the detection and proper analysis of ME cases.

\section{Recommendations}

Health care settings should maintain confidence with their staff to report patient safety incidents and near misses without fear of being blamed or punished. Educational programs for healthcare professionals and patients on efficient reporting of MEs are urgently needed to reduce MEs and improve patient care through educational training by clinical pharmacists. Furthermore, the medical team should pay attention to patients' common complains, so that they would take necessary precautions to prevent their reoccurrence. Routine auditing by health authorities and internal monitoring systems in hospitals should be regularly enforced.

Maintaining case Follow up with the reporters/ patients is of great importance for the reported data to be complete and accurate to allow for proper case assessment, learning from the reported errors, and avoiding ME risks.

Further studies on a large scale are recommended.

\section{Conflict of interest}

The authors declare that they have no conflicts of interest.

\section{Funding statement}

Not applicable

\section{REFERENCES}

1. Makary MA, Daniel M. Medical Error - The Third Leading Cause of Death in the U.S. BMJ 2016; 353: i2489 doi: https://doi.org/10.1136/bmj.

2. Institute of Medicine, Committee on Identifying and Preventing Medication Errors, Preventing Medication Errors. Washington (DC): National Academies Press 2007; 12425.

3. Wittich CM, Burkle CM, Lanier WL. Medication errors: an overview for clinicians. Mayo Clin Proc 2014; 89(8):1116-25. doi: 10.1016/j.mayocp.2014.05.007. Epub 2014 Jun 27.

4. Institute of Medicine (US) Committee on Quality of Health Care in America; Kohn LT, Corrigan JM, Donaldson MS, editors. To Err is Human: Building a Safer Health System. Washington (DC): National Academies Press (US); 2000. Available from: https://www.ncbi.nlm.nih.gov/books/NBK 225182/ doi: 10.17226/9728

5. Whittaker CF, Miklich MA, Patel RS, Fink JC. Medication Safety Principles and Practice in CKD. Clin J Am Soc Nephrol 2018 Nov 07; 13(11):1738-1746.

6. European Medicines Agency. European Union regulatory workshop on medication errors. EMA/144458/2013. Available from: https://www.ema.europa.eu/docs/en_GB/.../W C500143163.pdf Last accessed 2/7/2019 
7. Ferner RE, Aronson JK. Clarification of terminology in medication errors: Definitions and Classification. Drug Saf 2006; 29(11):1011-22.

8. Managing Drug Supply (MDS-3): Managing Access to Medicines and Health Technologies. Part II: Pharmaceutical ManagementChapter(35): Pharmacovigilance. 3rd ed. Management Sciences for health; 2012. Available from: http://apps.who.int/medicinedocs/en/m/abs tract/Js19577en/

9. Anderson JG, Abrahamson K. Your Health Care May Kill You: Medical Errors. Stud Health Technol Inform. 2017; 234: 13-17. DOI 10.3233/978-1-61499-742-9-13

10. Pham JC, Story JL, Hicks RW, Shore AD, Morlock LL, Cheung DS, et al. National study on the frequency, types, causes, and consequences of voluntarily reported emergency department medication errors. J Emerg Med 2011;40: 485-492.

11. World Health Organisation. Reporting and learning systems for medication errors: the role of pharmacovigilance centers. Geneva: WHO Press; 2014.

12. Alj L, Touzani MDW, Benkirane R, Edwards IR, Soulaymani R. Detecting medication errors in pharmacovigilance database: capacities and limits. Int J Risk Saf Med. 2007; 19(4): 187-94.

13. Milch C, Salem D, Pauker S, Lundquist T, Kumar S, Chen J. Voluntary electronic reporting of medical errors and adverse events: an analysis of 92,547 reports from 26 acute care hospitals. J Gen Intern Med 2006; 21:165-170.

14. Hibbs BF, Moro PL, Lewis P, Miller ER, Shimabukuro TT. Vaccination errors reported to the Vaccine Adverse Event Reporting System, (VAERS) United States, 2000-2013. (2015) Jun 22; 33(28): 3171- 3178. doi: 10.1016/j.vaccine.2015.05.006

15. Benjamin DM. Reducing medication errors and increasing patient safety: case studies in clinical pharmacology. J Clin Pharmacology 2003; 43: 768-83.

16. Guideline on the Regulation of Therapeutic Products in New Zealand, New Zealand Medicines and Medical Devices Safety Authority - Part 8 - Edition 2.1.(2017). https://www.medsafe.govt.nz/regulatory/Gui deline/GRTPNZ/part-8-

pharmacovigilance.pdf

17. Wettach C, Thomann J, Lambrigger-Steiner C, Buclin T, Desmeules J, von Mandach U. Pharmacovigilance in pregnancy: adverse drug reactions associated with fetal disorders. J Perinat Med. 2013; 41(3):301-7.

18. Anderson C, Gifford A, Avery A, Fortnum H, Murphy E, Krska J, Bond C. Yellow Card Study Collaboration. Assessing the usability of methods of public reporting of adverse drug reactions to the UK Yellow Card Scheme. Health Expect. 2012; 15(4): 433- 40. doi: 10.1111/j.1369-7625.2011.00686.x. Epub 2011 Apr 27. PubMed PMID: 21521431; PubMed Central PMCID: PMC5060635. 